\title{
Unintentional weight loss in older adults
}

\author{
Svetlana Stajkovic MD, Elizabeth M. Aitken BSc MLIS, Jayna Holroyd-Leduc MD
}

\section{The case}

A 73-year-old woman presents to your clinic complaining of unintentional weight loss. She reports having lost $15 \mathrm{lbs}(6.8 \mathrm{~kg})$ over the past year. Previously, her weight was $135 \mathrm{lbs}(61.3 \mathrm{~kg})$ and now it is $120 \mathrm{lbs}(54.5 \mathrm{~kg})$. She reports that she is eating three meals per day as usual.

The patient's past medical history is notable for osteoporosis, a left hip fracture three years ago, osteoarthritis, hypothyroidism and hypercholesterolemia. She is taking levothyroxine, a statin and a bisphosphonate plus vitamin D and calcium. She recently started taking nonsteroidal anti-inflammatory medications (NSAIDs) as needed for knee pain. She is an exsmoker and does not drink alcohol. On examination, her weight was $121 \mathrm{lbs}(55 \mathrm{~kg})$, with a body mass index (BMI) of 22. She also had osteoarthritic changes in the knees. Results of investigations, including radiograph of the chest, complete blood count, electrolytes, creatinine, thyroid stimulating hormone and albumin are within normal limits.

$\mathrm{U}$ nintentional or involuntary weight loss is a common phenomenon among older adults, with an annual incidence of approximately $13 \% .^{1}$ Problematic weight loss in the older adult is defined by the United States Omnibus Budget Reconciliation Act of 1987 (Title IV: subtitle C: Nursing Home Reform) as a loss of $5 \%$ of body weight in one month or $10 \%$ over a period of six months or longer. ${ }^{2,3}$ In this review, we focus on unintentional weight loss for which no organic cause can be found, although frequently, the loss of weight may be associated with chronic conditions. ${ }^{3}$ Older patients who involuntarily lose substantial amounts of weight without an obvious cause can pose difficult diagnostic and management dilemmas for physicians.

The clinical consequences of involuntary weight loss include functional decline, infections, decubitus ulcers, exacerbation of cognitive and mood disorders, and increased use of acute and long-term care facilities. ${ }^{4}$ Mechanisms for involuntary weight loss can include decreased intake, accelerated metabolism and increased caloric loss in urine or stool. ${ }^{5}$
Inflammatory cytokines, including tumour necrosis factor- $\alpha$ (TNF- $\alpha$ ), interleukin- $1 \beta$ and interleukin- 6 have been implicated in cachexia and weight loss. ${ }^{1}$ Historically termed cachectin, TNF- $\alpha$ is considered one of the more prominent cytokines and is thought to be a primary mediator of the muscle wasting of cachexia; it is also believed to act synergistically with interleukin$1 \beta$ to promote cachexia. ${ }^{1}$ Cytokines may act both centrally, by inhibiting feeding behaviour, and peripherally, by decreasing gastric motility, gastric emptying and intestinal motility and by modifying gastric secretion. ${ }^{1}$ Tumour necrosis factor- $\alpha$ levels are elevated in several human disease states associated with cachexia and weight loss, including malignancy, acquired immunodeficiency syndrome, heart failure, rheumatoid arthritis and chronic obstructive pulmonary disease.

Given that unintentional weight loss is a common condition among older adults and is associated with adverse outcomes, our objective was to review the evidence regarding risk factors, differential diagnosis, prognosis, investigation and treatment of unintentional weight loss in this population. In this review, we use the term "unexplained weight loss" to refer to unintentional weight loss for which there is no specific organic cause.

\section{Literature review}

Searches of MEDLINE (and MEDLINE InProcess), EMBASE, CINAHL and AGELINE were conducted to identify relevant studies from 1980 to September 2009. Additional studies

\section{- Ker points}

- Medications, functional limitations, intake issues, psychological problems, reduced social activity and low income can all contribute to unexplained weight loss.

- A comprehensive history and physical examination has the greatest potential for eliciting the cause(s) of weight loss.

- Computed tomography screening is of limited value. Instead, diagnostic testing should be directed toward areas of concern based on the history and physical examination.

- There is minimal evidence to support use of specific nutritional interventions or pharmacologic agents.
Competing interests: None declared.

Disclaimer: Jayna HolroydLeduc is associate editor of Reviews for $C M A J$ and was ot involved in the editorial decision-making process for this article.

This article has been peer reviewed.

Correspondence to: jayna.holroyd-leduc @albertahealthservices.ca

(10.1503 /cmaj.101471 
were identified from searching bibliographies of retrieved articles and by consulting a clinical expert in the area. We identified Englishlanguage articles that addressed risk factors, differential diagnosis, prognosis, investigation or treatment of unintentional weight loss among adults 65 years of age or older. Further details on the search can be found in Appendix 1, available at www.cmaj.ca/cgi/content/full/cmaj.101471 /DC1. We excluded articles that specifically focused on weight loss associated with cancer or HIV infection. All types of articles were considered for inclusion except for case reports, editorials and meeting abstracts. All patients, regardless of where they lived, were included in the review. Two reviewers (S.S. and E.M.A or J.H-L) independently reviewed all identified citations to select relevant publications that met the inclusion criteria. In cases of doubt, full-text articles were retrieved for review and discussion.

Two investigators (S.S. and E.M.A or J.H-L) then independently reviewed all retrieved fulltext articles to confirm inclusion criteria were met. A third reviewer (S.S., E.M.A or J.H-L) resolved disagreements. Using a standardized data abstraction sheet, information was extracted that was pertinent to the study design, study population and size, clinical setting, outcomes and results. A meta-analysis was not conducted because of clinical heterogeneity among included articles. Instead, a summary of data is presented.

The search identified 726 potential citations. Of these, 198 full-text articles were reviewed, and 30 studies met criteria for inclusion (Appendix 1).

\section{What factors are associated with unexplained weight loss?}

The risk factors for weight loss in the older adult can be classified into the following three main categories: physiologic factors (e.g., chronic and acute diseases), psychological factors (e.g., depression, bereavement) and social factors (e.g., isolation, social problems). ${ }^{6}$

\section{Physiologic factors}

Based on evidence from a large cohort study that involved 4010 persons aged 65 years and older from 11 cities in Europe, the most common independent factors associated with unexplained weight loss are those related to food intake. Specifically, they are eating less than one meal per day (odds ratio [OR] 4.2, 95\% confidence interval [CI] 2.8-6.4), eating less overall (OR 2.8, 95\% CI 1.8-4.4), reduced appetite (OR 2.5, 95\% CI 1.9-3.4), severe malnutrition (OR 7.1, 95\% CI 4.2-11.9) and problems swallowing food (OR
$2.8,95 \%$ CI $1.8-4.4)$. Other factors were flareups of chronic diseases (OR 1.5, 95\% CI 1.12.1), hospital admission in the last 90 days (OR 2.1, 95\% CI 1.6-2.7), constipation (OR 1.9, 95\% CI 1.3-2.7), falls (OR 1.5, 95\% CI 1.2-1.9), pressure ulcers (OR $1.5,95 \%$ CI 1.2-1.9) and daily pain (OR 1.3, 95\% CI 1.0-1.6) (Box 1).

A retrospective chart review of 96 residents in six intermediate care facilities in the United States found an association between anorexia and poor weight status and confusion in 42 residents. ${ }^{8}$ A prospective six-month study involving 309 residents of an intermediate nursing home in suburban midwestern United States found the primary reasons for weight changes to be acute illness, dementia and changes in the mucous membranes of the mouth and gums. ${ }^{9}$

A retrospective chart review in a 718-bed long-term care facility in the eastern United States, where 41 residents ( 24 women with a mean age of 80 years) had recently lost weight

Box 1: Factors associated with unexplained weight loss

\section{Physiologic factors}

- Disease-related issues ${ }^{7-9}$

- Acute illness

- Recent admission to hospital (i.e., in last three months)

- Flare-up of chronic disease

- Dementia or confusion

- Constipation

- Pressure ulcers

Daily pain

- Medication-related issues ${ }^{2}$

- Functional issues $3,7,10$

- Fluctuating or reduced function

- Compromised mobility

Falls

- Intake-related issues ${ }^{7,9,10}$

- Eating, chewing or swallowing problems

- Poor oral health

- Reduced appetite

Low or reduced food intake

Thirst

- Laboratory values ${ }^{3}$

- Serum albumin < 35 g/L with normal hydration

Total cholesterol $<4.2 \mathrm{mmol} / \mathrm{L}$

Psychological factors ${ }^{9,12}$

- Depression

- Bereavement

Social factors ${ }^{7,8}$

- Reduced social activity

- Low income 
found that more than $75 \%$ of them had been prescribed a medication that could potentially contribute to weight loss (Box 2). ${ }^{2}$ However, when reviewing possible causes of weight loss, no physicians changed medications in an attempt to reverse this problem. Instead, many ordered additional medications to help stimulate appetite.

Among a cohort of 250 residents of a Dutch nursing home, after adjusting for age and sex, a significant relationship was seen between body weight and mobility $(p<0.0001)$, appetite $(p<0.001)$, thirst $(p<0.01)$ and consumption of extra food $(p<0.0001){ }^{10}$ In multivariate analysis, only difficulties in bringing food to the mouth and chewing were significantly associated with weight loss. Similarly, in a cross-sectional study involving 109 patients (99\% male) admitted to a geriatric rehabilitation unit in the United States, oral problems were the strongest predictor of substantial, involuntary weight loss in the year before admission. ${ }^{11}$

A chart review of 290 medical records from many centres in the United States that included long-term care residents and home care clients found six factors to be associated with unexplained weight loss. ${ }^{3}$ These factors included reduced functional ability, taking in $50 \%$ or less of the food served in three consecutive days, refusal of $50 \%$ or more of food replacement offered over a seven-day period, chewing problems, a serum albumin level less than $35 \mathrm{~g} / \mathrm{L}$ with normal hydration status and a cholesterol level less than $4.1 \mathrm{mmol} / \mathrm{L}$.

\section{Psychological factors}

A cross-sectional study of 68 communitydwelling older adults in the midwestern United States (with an average age of 86 years and 51 of whom were women) showed that depression (using the Geriatric Depression Scale) was independently associated with weight loss (OR 1.65, 95\% CI 1.12-2.43). ${ }^{12}$ Another prospective study $(n=309)$ found that psychiatric and psychological diseases are one of the primary reasons for unexplained weight loss. ${ }^{9}$

\section{Social factors}

A large cohort study $(n=4010)$ found that reduced social activity is an independent contributor to unexplained weight loss in the older person (OR 2.0, 95\% CI 1.6-2.5). ${ }^{7}$ Additionally, a retrospective chart review of 96 residents in six intermediate care facilities in the United States showed that unexplained weight loss was most prevalent among the lowest income group. ${ }^{8}$

In summary, determination of potential contributing factors to weight loss should not focus solely on disease-related processes. Functional issues, medications, issues with intake, and psychological and social factors also need to be considered.

\section{How should a patient with unintentional weight loss be assessed?}

A chart review of 10000 patients in seven family practice centres in the southeastern United States, where 45 patients (with an average age of 72 years and 30 of whom were women) were identified as having substantial, unexplained weight loss, showed that a comprehensive history and physical examination have the greatest potential for eliciting the cause or causes of weight loss. ${ }^{5}$ Information about potential physiologic, psychological and social factors (e.g., dementia, immobility, bereavement, low income, social isolation) may prove vital to determining the cause as well as to the outcome (Box 1). All current prescribed and over-the-counter medications should be reviewed. ${ }^{2}$

Although testing should be directed toward areas of concern based on the history and physical examination, tests found to be of highest yield in identifying potential causes include stool hemoccult, barium enema, sigmoidoscopy, upper gastrointestinal series, endoscopy and thyroid function tests. ${ }^{5}$ Computed tomographic screening offers no further information. ${ }^{5}$ In institutionalized older adults, hemoglobin level, total cholesterol and albumin are useful in making the diagnosis. ${ }^{13}$ There are a few studies that have looked at TNF- $\alpha$ levels, ${ }^{1}$ cerebrospinal fluid concentration of amino acids, ${ }^{14}$ plasma and cerebrospinal fluid cytokine levels, ${ }^{15}$ and plasma and cerebrospinal fluid neuropeptide levels. ${ }^{16}$ However, these levels have limited value within routine clinical practice.

In summary, a comprehensive history together with a physical examination should be the first step in eliciting the cause or causes of the weight loss. This step includes screening for potential risk factors and assessing current medications. Computed tomographic screening is of limited value. Instead, diagnostic testing should be directed toward areas of concern based on the history and physical examination.

\section{How can unexplained weight loss be treated?}

Treatment needs to be individualized and targeted to the patient's risk factors and to findings on history, physical examination and investigations. 


\section{Nonpharmacologic interventions}

Four studies looked at nutritional interventions or nutritional interventions combined with exercises (Table 1). ${ }^{17-20}$ All four studies were randomized trials but three were small $(n<100))^{17,18,20}$ Only one trial was blinded ${ }^{17}$ and only one used intention-to-treat analysis. ${ }^{18}$

A randomized, double-blind trial conducted in Denmark among 24 hospitalized patients compared differences in total nutrient intake between a diet that included a standard $(4.2 \mathrm{~kJ} / \mathrm{ml})$ commercial liquid supplement and a diet incorporating a nutrient-dense $(6.3 \mathrm{~kJ} / \mathrm{ml})$ supplement. ${ }^{17}$ The products were offered in addition to the regular hospital diet. Both diets increased total daily energy and protein intake, with no significant difference between the diets. Poor compliance, occurring in one-third to one-half of patients, was evident during the first two to three days of supplementation.

Another randomized crossover study $(n=34)$ looked at the provision of breakfast food for dinner to patients with dementia, given that breakfast food is generally well consumed. ${ }^{20}$ Consumption of nutritional supplements between breakfast and dinner was also encouraged. There was no significant change in mean body weight with the intervention.

A third study examined the impact of physical training and nutrition. ${ }^{18}$ Fifty-eight older, community-dwelling individuals were randomized to one of the following four groups: a physical training program (involving aerobic, muscle strength and balance training), a nutritional intervention program (involving individually targeted advice and group sessions), a combination of the first two interventions, or a control group. At baseline and then again at 12 weeks, subjects were screened for physical performance. Intention-to-treat analysis showed a significant improvement in both training groups compared with the nutritional group. The nutritional interventions showed no significant improvement over the control group.

Another prospective trial randomized 29 dieticians to the provision of usual nutritional care or a new medical nutritional therapy protocol for prevention and treatment of unexplained weight loss among long-term care residents. ${ }^{19}$ The new protocol emphasized assessment; intervention (including weighing frequency); communication with staff, medical doctor, family and resident; and reassessment. Fourteen out of 364 residents (4\%) admitted with significant preexisting weight loss were successfully treated within 90 days after admission. Dieticians in both groups were equally successful at treating pre-existing weight loss when it was identified. Differences were found in nutritional care activi- ties. Dieticians providing the new protocol reported more nutritional assessment activities, whereas dieticians providing usual care reported more interventional activities.

\section{Pharmacologic treatment}

Four trials (10 publications) examined pharmacologic treatment options for unexplained weight loss (Table 2). ${ }^{14-16,21-27}$ All four trials were small and only one was a blinded randomized control trial. ${ }^{23-27}$ The most commonly studied medication was megestrol acetate. One study also looked at the use of dronabinol for unexplained weight loss.

In one blinded randomized control trial (five publications), megestrol acetate was used in the treatment of unexplained weight loss. ${ }^{23-27}$ Sixtynine patients were randomly assigned to receive placebo or megestrol $800 \mathrm{mg} / \mathrm{d}$ for 12 weeks. At 12 weeks, there were no significant differences in weight gain between treatment groups, although patients treated with megestrol reported significantly greater improvements in appetite, enjoyment of life and well-being. There was no difference in survival between the groups at four years.

A prospective trial in four long-term care facilities examined the role of megestrol acetate and optimal feeding assistance. ${ }^{21}$ For 63 days, megestrol $(400 \mathrm{mg} / \mathrm{d})$ was given to 17 residents who were eating less than $75 \%$ of most meals. They received either usual care or optimal feeding assistance. Results suggest that megestrol in combination with optimal mealtime feeding assistance significantly increased oral intake in frail long-term care residents but was not effective under usual care conditions.

One small case-control study (three publications) compared 14 patients who had anorexia with 10 control patients. ${ }^{14-16}$ The patients with anorexia had a median age of 78 (standard deviation [SD] 8) years and BMI of 18.4 (SD 0.6) $\mathrm{kg} / \mathrm{m} 2$ in the absence of any organic or mental disorders. Data from the control group were collected retrospectively by reviewing 24 hospital records of persons over 65 years of age in whom a lumbar puncture had been performed to rule out a meningeal syndrome. The 10 patients in the control group were selected because they were nonsmokers and had a normal body weight (i.e., within $10 \%$ of their ideal body weight). The study primarily focused on examining the changes in anthropometric parameters, amino acids, neuropeptides and cytokine levels associated with anorexia. Of the 14 anorectic patients, five received treatment with megestrol acetate (480 mg/day) for six months. There were no changes in anthropometric parameters with treatment. The only significant changes in laboratory 
parameters were an increase in plasma transferrin level $(p<0.05)$ and an increase in CSF $\beta$ endorphin levels $(p<0.05)$.

Common side effects of megestrol acetate include hypertension, gastrointestinal upset, insomnia and impotence; reported serious adverse events include adrenal insufficiency and thromboembolic events. ${ }^{28}$

One retrospective uncontrolled observational study of dronabinol was identified (starting dose $2.5 \mathrm{mg}$ nightly for one week; dose could be increased to $2.5 \mathrm{mg}$ twice daily $30 \mathrm{~min}$. before lunch and dinner at the discretion of the physician). ${ }^{22}$ Treatment showed a trend toward weight gain among the 28 long-term care residents, who were treated for 12 weeks. No controlled trials of dronabinol were identified; therefore, this medication cannot be recommended.

In summary, initial treatment for unexplained weight loss should be targeted at addressing identified risk factors, although evidence of benefit is limited. Medications that are not clearly required and that may be contributing to the weight loss should be discontinued or appropriate alternatives considered. The role for specific nutritional interventions targeted at increasing caloric intake and improving weight is unclear. There is also minimal evidence to support use of pharmacologic agents. Megestrol acetate may be effective for older adults living in care facilities when used in conjunction with feeding assistance, but further study is required.

\section{What is the prognosis for those with unintentional weight loss?}

Unintentional weight loss of more than $4 \%$ in a year appears to be an independent predictor of increased mortality (relative risk [RR] 2.43, 95\% CI 1.34-4.41). ${ }^{4}$ In a prospective study of 41836 women, conducted in the United States as part of the Iowa Women's Health Study, one or more episodes of unintentional weight loss of more than 20 pounds during adulthood was associated with a $46 \%-57 \%$ higher rate of death. ${ }^{29}$ A prospective study of 4869 male patients older than 65 years from general practices in 24 towns across the United Kingdom found that unintentional weight loss was associated with higher mortality risk only among those with cancer (adjusted relative risk [ARR] 1.71, 95\% CI 1.33-2.19) after adjustment for lifestyle characteristics and pre-existing disease. ${ }^{30} \mathrm{~A}$ retrospective chart review of 148 longterm care residents residing in the southeastern United States found that those who lost $5 \%$ or more of their body weight within one month were 4.6 times more likely to die within one year. ${ }^{31}$
Unintentional weight loss appears to be associated with an increased risk of death among both older adults living in care facilties and community-dwelling older adults. In general, the impact on life expectancy of treating the weight loss remains unclear.

Box 2: Examples of medications that can potentially cause weight loss ${ }^{2,32}$

Cardiac

- Digoxin

- Aspirin

- Angiotensin-converting enzyme inhibitors

- Calcium channel blockers

- Hydralazine

- Loop diuretics

- Hydrochlorothiazides

- Spironolactone

- Statins

- Nitroglycerin

Neurologic and psychiatric

- Selective serotonin reuptake inhibitors

- Tricyclics

- Neuroleptics

- Benzodiazepines

- Anticonvulsants

- Lithium

- Levodopa

- Dopamine agonists

- Donepezil

- Memantine

Bones and joints (including pain medications)

- Bisphosphonates

- Nonsteroidal anti-inflammatory drugs (including COX-2 inhibitors)

- Opiates

- Allopurinol

- Colchicine

- Gold

- Hydroxychloroquine

Endocrine

- Levothyroxine

- Metformin

Other

- Anticholinergics

- Antibiotics

- Decongestants

- Antihistamines

- Iron

- Potassium

- Alcohol

- Nicotine 


\section{Gaps in knowledge}

This review excluded $11 \%$ of the retrieved references because they were not written in English. The overall quality of the literature retrieved was low. As a result, there is insufficient evidence to support the routine use of either nutritional interventions or pharmacologic treatments to help address unintentional weight loss. High-quality, adequately powered trials are needed to determine what interventions are effective at addressing unintentional weight loss and the associated clinical outcomes, such as mortality.

\section{The case revisited}

On further questioning, the patient admits that even though she had been eating three meals per day, she eats less at each meal than previously. She tells you that her husband of 50 years died suddenly 10 months ago. She reports her mood is fine but that she still has not gotten over his death. She feels lonely and is finding it difficult to motivate herself to prepare adequate meals for only one person. She also reports experiencing nausea and some difficulty chewing over the past month. You take a closer look in her mouth and notice that her dentures are loose and that there are a few small ulcers on her hard palate.

In an attempt to address her risk factors, you advise her to have her dentures adjusted. Suspecting that the NSAIDS may be contributing to her nausea, you advise her to use acetaminophen for her knee pains instead. At your encouragement, she starts attending grief counselling and becomes involved in social activities, including a supper club, at her local seniors centre.

Over the next two months, her appetite improves and she gains four pounds. Her weight loss appears to have been the result of multiple factors, including social isolation, bereavement, chewing issues, decreased oral intake and possibly the use of NSAIDs. Risk factor modification appears to have been successful so you do not consider further nutritional or pharmacologic interventions at this time.

\section{Conclusion}

There are many factors associated with unintentional weight loss. Assessment of unintentional weight loss should start with a comprehensive history, including questions about associated factors, and a physical examination. Investigations should be guided by the findings of the history and physical examination. Both nutritional and pharmacologic interventions have proven so far to be of only limited value. Although treatment remains a challenge, clinicians should attempt to identify and address factors that may be contributing to the weight loss.

\section{References}

1. Ruscin JM, Page RL II, Yeager BF, et al. Tumor necrosis factoralpha and involuntary weight loss in elderly, communitydwelling adults. Pharmacotherapy 2005;25:313-9.

2. Goldeberg RJ, Kaplan LA, Boucher LJ. Physicians' attentiveness to medication use as etiology of weight loss. Long term care interface 2005;6:20-3.

3. Gilmore SA, Robinson G, Posthauer ME, et al. Clinical indicators associated with unintentional weight loss and pressure ulcers in elderly residents of nursing facilities. J Am Diet Assoc 1995;95:984-92.

4. Wallace JI, Schwartz RS, LaCroix AZ, et al. Involuntary weight loss in older outpatients: incidence and clinical significance. $J$ Am Geriatr Soc 1995;43:329-37.

5. Thompson MP, Morris LK. Unexplained weight loss in the ambulatory elderly. J Am Geriatr Soc 1991;39:497-500.

6. Satheannoppakao W. Risk factors associated with predicting involuntary weight loss among elderly nursing home residents. Corvallis (OR): Oregon State University; 2004.

7. Sorbye LW, Schroll M, Finne Soveri H, et al. Unintended weight loss in the elderly living at home: the aged in Home Care Project (AdHOC). J Nutr Health Aging 2008;12:10-6.

8. Bartlett BJ. Characterization of anorexia in nursing home patients. Educ Gerontol 1990;16:591-600.

9. Wright BA. Weight loss and weight gain in a nursing home: a prospective study. Geriatr Nurs 1993;14:156-9.

10. Berkhout AM, Cools HJ, van Houwelingen HC. The relationship between difficulties in feeding oneself and loss of weight in nursing-home patients with dementia. Age Ageing 1998;27:637-41.

11. Sullivan DH, Martin W, Flaxman N, et al. Oral health problems and involuntary weight loss in a population of frail elderly. $J \mathrm{Am}$ Geriatr Soc 1993;41:725-31.

12. Callen BL, Wells TJ. Screening for nutritional risk in community-dwelling old-old. Public Health Nurs 2005;22:138-46.

13. Chen LK, Lin MH, Hwang SJ, et al. Nutritional status and clinical outcomes among institutionalized elderly Chinese in Taiwan. Arch Gerontol Geriatr 2007;44:315-23.

14. Martinez M, Arnalich F, Vazquez JJ, et al. Altered cerebrospinal fluid amino acid pattern in the anorexia of aging: relationship with biogenic amine metabolism. Life Sci 1993;53:1643-50.

15. Martinez M, Arnalich F, Hernanz A. Alterations of anorectic cytokine levels from plasma and cerebrospinal fluid in idiopathic senile anorexia. Mech Ageing Dev 1993;72:145-53.

16. Martinez M, Hernanz A, Gomez-Cerezo J, et al. Alterations in plasma and cerebrospinal fluid levels of neuropeptides in idiopathic senile anorexia. Regul Pept 1993;49:109-17.

17. Ovesen L. The effect of a supplement which is nutrient dense compared to standard concentration on the total nutritional intake of anorectic patients. Clin Nutr 1992;11:154-7.

18. Rydwik E, Lammes E, Frandin K, et al. Effects of a physical and nutritional intervention program for frail elderly people over age 75. A randomized controlled pilot treatment trial. Aging Clin Exp Res 2008;20:159-70

19. Splett PL, Roth-Yousey LL, Vogelzang JL. Medical nutrition therapy for the prevention and treatment of unintentional weight loss in residential healthcare facilities. J Am Diet Assoc 2003; 103:352-62.

20. Wei Hing Young K. Responses to nutrition interventions aimed at increasing food intake in seniors with Alzheimer's disease and their associations with body mass index, cognitive impairment and behavioral difficulties. Toronto (ON): University of Toronto; 2004.

21. Simmons SF, Walker KA, Osterweil D. The effects of megestrol acetate on oral food and fluid intake in nursing home residents: a pilot study. J Am Med Dir Assoc 2004;5:24-30.

22. Wilson MM, Philpot C, Morley JE. Anorexia of aging in long term care: is dronabinol an effective appetite stimulant? - a pilot study. J Nutr Health Aging 2007;11:195-8.

23. Yeh S, Wu SY, Levine DM, et al. Quality of life and stimulation of weight gain after treatment with megestrol acetate: correlation between cytokine levels and nutritional status, appetite in geriatric patients with wasting syndrome. J Nutr Health Aging 2000; 4:246-51.

24. Yeh SS, Hafner A, Chang CK, et al. Risk factors relating blood markers of inflammation and nutritional status to survival in cachectic geriatric patients in a randomized clinical trial. $J$ Am Geriatr Soc 2004;52:1708-12. 
25. Yeh SS, Lovitt S, Schuster MW. Usage of megestrol acetate in the treatment of anorexia-cachexia syndrome in the elderly. $J$ Nutr Health Aging 2009:13:448-54.

26. Yeh SS, Wu SY, Lee TP, et al. Improvement in quality-of-life measures and stimulation of weight gain after treatment with megestrol acetate oral suspension in geriatric cachexia: results of a double-blind, placebo-controlled study. J Am Geriatr Soc 2000;48:485-92.

27. Yeh SS, Wu SY, Levine DM, et al. The correlation of cytokine levels with body weight after megestrol acetate treatment in geriatric patients. J Gerontol A Biol Sci Med Sci 2001;56:M48-54.

28. Megestrol acetate: adverse effects. In: DrugPoints System Micromedex Healthcare Series [Internet database]. Greenwood Village (CO): Thomson Reuters (Healthcare) Inc. Updated periodically. Available: www.thomsonhc.com/hcs/librarian (accessed 2011 Jan. 27).

29. French SA, Folsom AR, Jeffery RW, et al. Prospective study of intentionality of weight loss and mortality in older women: the Iowa Women's Health Study. Am J Epidemiol 1999;149: 504-14.

30. Wannamethee SG, Shaper AG, Lennon L. Reasons for intentional weight loss, unintentional weight loss, and mortality in older men. Arch Intern Med 2005;165:1035-40.

31. Ryan C, Bryant E, Eleazer P, et al. Unintentional weight loss in long-term care: predictor of mortality in the elderly. South Med J 1995;88:721-4

32. Alibhai SM, Greenwood C, Payette H. An approach to the management of unintentional weight loss in elderly people. CMAJ $2005 ; 172: 773-80$

Affiliations: From the Department of Medicine (Stajkovic), the University Library (Aitken) and the Departments of Medicine and Community Health Sciences (Holroyd-Leduc), University of Calgary, Calgary, Alta.

Contributors: Svetlana Stajkovic and Jayna Holroyd-Leduc developed the concept of the review. Elizabeth Aitken conducted the literature search. All of the authors reviewed and critically appraised the literature cited in the manuscript. Svetlana Stajkovic drafted the article, and all of the authors revised the manuscript critically for important intellectual content and approved the final version submitted for publishing.

\section{Still sharp in} your fight against hypertension.

\author{
$\checkmark$ Powerful BP-lowering1 \\ $\checkmark$ 24-hour BP control demonstrated with ACCUPRIL ${ }^{1}$ \\ $\checkmark$ The flexibility of seven dosage strengths \\ with ACCUPRIL and ACCURETIC ${ }^{1,2}$ \\ $\checkmark$ All dosages at a single flat price ${ }^{3 \dagger}$
}

\section{ACCUPRIL \\ TAKE A FORWARD STEP}

ACCUPRIL is indicated in essential hypertension when diuretics or beta-blockers are unsuitable.

WARNINGS: As with all ACE inhibitors, please refer to specific wamings regarding drug discontinuation in angioedema and pregnancy.

The most frequent adverse events in controlled clinical trials with ACCUPRIL were headache $(8.1 \%)$, dizziness $(4.1 \%)$, cough $(3.2 \%)$, fatigue $(3.2 \%)$, rhinitis (3.2\%), nausea and/or vomiting (2.3\%) and abdominal pain $(2.0 \%)$. For the complete list of adverse events, please refer to the Product Monograph. ACCURETIC is indicated in essential hypertension when combination therapy is appropriate. The fixed combination is not for initial therapy.

Quinapril is contraindicated in pregnancy. ACE inhibitors can cause fetal and neonatal morbidity and mortality when administered to pregnant women. Several dozen cases have been reported in the world literature. Quinapril should not be used by women who are pregnant, intend to become pregnant, or could become pregnant and who are not using adequate contraceptive measures. It is possible that quinapril passes into breast milk. Patients should be advised not to breast-feed while taking quinapril. See prescribing information for complete contraindications. When used in pregnancy, angiotensin converting enzyme (ACE) inhibitors can cause injury or even death of the developing fetus. When pregnancy is detected ACCUPRIL or ACCURETIC should be discontinued as soon as possible.

The most frequent adverse events in controlled trials with ACCURETIC were headache $(6.7 \%)$, dizziness $(4.8 \%)$, cough $(3.2 \%)$ and fatigue $(2.9 \%)$. For the complete list of adverse events, please refer to the Product Monograph.

\section{Pfizer}

อ 2011 Pfizer Canada Inc.

Kirkland, Quebec H9J 2M5

ACCUPRIL ${ }^{\oplus}$ and ACCURETIC ${ }^{\oplus}$

Parke, Davis \& Company LLC, owner

Pfizer Canada Inc., Licensee

Im Pfizer Inc., owner/

Pfizer Canada Inc. Licensee

† Price does not include pharmacy professional fees. Please refer to Product Monographs for complete dosing information.

(RED $\overline{\text { PAAB }}^{\text {Member }}$

See prescribing summary on page 498 